\title{
Symptomatic Narcolepsy and Generalized Seizures Following Surgery for Rathke's Cleft Cyst
}

\author{
On-Hwa Ryu, MD, Han Uk Ryu, MD, Sang-Ahm Lee, MD \\ Department of Neurology, Asan Medical Center, University of Ulsan College of Medicine, Seoul, Korea
}

\begin{abstract}
Symptomatic narcolepsy is a rare disorder caused by other underlying neurological disorders. One of the most frequent causes for symptomatic narcolepsy is brain tumors, usually in the region of the third ventricle and the diencephalon. Depending on clinical profile, the mass effect or surgical removal of space occupying lesions may play a definitive causative role in the occurrence of narcolepsy symptoms. We report a woman with symptomatic narcolepsy that developed 2 years after surgical removal of Rathke's cleft cyst, which has never before been reported as a causative factor for symptomatic narcolepsy. In addition, myoclonic and/or generalized tonic-clonic seizures were associated with a left thalamic lesion generated by a complication of surgery.

Sleep Med Res 2014;5(2):72-76
\end{abstract}

Key Words Symptomatic narcolepsy, Hypothalamus, Generalized seizures, Rathke's cleft cyst.

Received: November 26, 2014

Revised: December 19, 2014

Accepted: December 29, 2014

Correspondence

Sang-Ahm Lee, MD

Department of Neurology,

Asan Medical Center,

University of Ulsan College of Medicine,

88 Olympic-ro 43-gil, Songpa-gu,

Seoul 138-736, Korea

Tel +82-2-3010-3445

Fax +82-2-474-4691

E-mailssalee@amc.seoul.kr

\section{INTRODUCTION}

Narcolepsy is a chronic sleep disorder characterized by excessive daytime sleepiness and rapid eye movement (REM) sleep-related symptoms such as cataplexy, sleep paralysis, and hypnagogic hallucination. Animal studies show that genetic alterations in either hypocretin ligands or hypocretin receptor-2 can cause narcolepsy in mice and dogs. In humans, mutations in hypocretin-related genes appear to be rare, however, a loss of hypocretin-producing neurons has been demonstrated in human pathology. Consequently, patients with narcolepsy have low or no detectable hypocretin in their cerebrospinal fluid (CSF). ${ }^{1}$ Narcolepsy is strongly associated with the human leukocyte antigen DQB1*0602, suggesting the involvement of an autoimmune process in idiopathic narcolepsy. ${ }^{2}$

Symptomatic narcolepsy is a rare disorder secondary to the underlying neurological disorder. In this disorder, the signs and symptoms of narcolepsy are temporally associated with the underlying neurological process. One of the most frequent causes of symptomatic narcolepsy is brain tumors, usually in the region of the third ventricle and the diencephalon, including adenoma, angioma, astrocytoma, cerebral sarcoidosis, choroid plexus carcinoma, colloid cyst, craniopharyngioma, glioma, germinoma, glioblastoma, histiocytosis, lymphoma, medulloblastoma, and subependymoma. ${ }^{3}$ Abnormalities in sleep and wakefulness that can develop after surgical removal of tumors in the hypothalamic area seem to vary from fragmented nocturnal sleep without daytime sleepiness to typical narcolepsy with cataplexy. ${ }^{4}$ Tachibana et al. ${ }^{5}$ reported a series of craniophayringioma patients diagnosed with narcolepsy after surgery.

We report a woman with symptomatic narcolepsy that developed after surgical removal of Rathke's cleft cyst, which has never before been reported as a causative factor for symptomatic narcolepsy. Excessive daytime sleepiness and sleep onset REM sleep episodes were demonstrated using the multiple sleep latency test (MSLT). In addition, the patient also had generalized seizures, including myoclonic and/or tonic-clonic seizures, which are rarely comorbid in patients with symptomatic narcolepsy. 


\section{CASE REPORT}

A 15-year-old girl was admitted to our hospital for suprasellar cystic tumor and suspicious panhypopituitarism (Oct 2008). Generalized convulsions with loss of consciousness developed while she was watching television. On evaluation at the local hospital, a $12 \mathrm{~mm}$-sized tumor compressing the pituitary gland with suprasellar extension was found on brain MRI (Fig. 1). The patient had been in good health before the admission and her family history was non-significant. Her vital signs and neurologic examination were normal. Tumor resection was performed through transsphenoidal approach. Rathke's cleft cyst was diagnosed by pathological examination that demonstrated protein debris without calcification and keratin pearls.

She began to complain of an excessive amount of voiding accompanied by thirst and a tendency for sleepiness a few days after surgery. Using the cocktail test, multiple pituitary hor- mone deficiencies were observed. She was discharged with growth hormone $4 \mathrm{IU} /$ day, desmopressin $0.4 \mathrm{mg} /$ day, medroxyprogesterone $5 \mathrm{mg} /$ day, estropipate $0.625 \mathrm{mg} /$ day, methimazol $5 \mathrm{mg} / \mathrm{day}$, prednisolone $5 \mathrm{mg} /$ day for hormone therapy. Thirst was improved but the sleepiness remained. Two months after surgery, brain MRI showed a focal hyper signal intensity lesion in the anterior-superior-medial part of her left thalamus, suggesting cerebral infarction as a sequelae of surgery (Fig. 2).

Antiepileptic drugs such as valproic acid were discontinued one month after surgery. Although antiepileptic drugs were not administered, seizures did not recur until four months after the tumor resection. In February 2009, myoclonic jerks developed in proximal extremities and were sometimes associated with loss of consciousness as well as generalized tonic-clonic seizures. In June 2009, the frequency of the generalized tonicclonic seizures increased to several times a month. Electroencephalography (EEG) showed frequent frontal dominant 3-4 $\mathrm{Hz}$ generalized spike and wave complexes (Fig. 3). Levetirace-
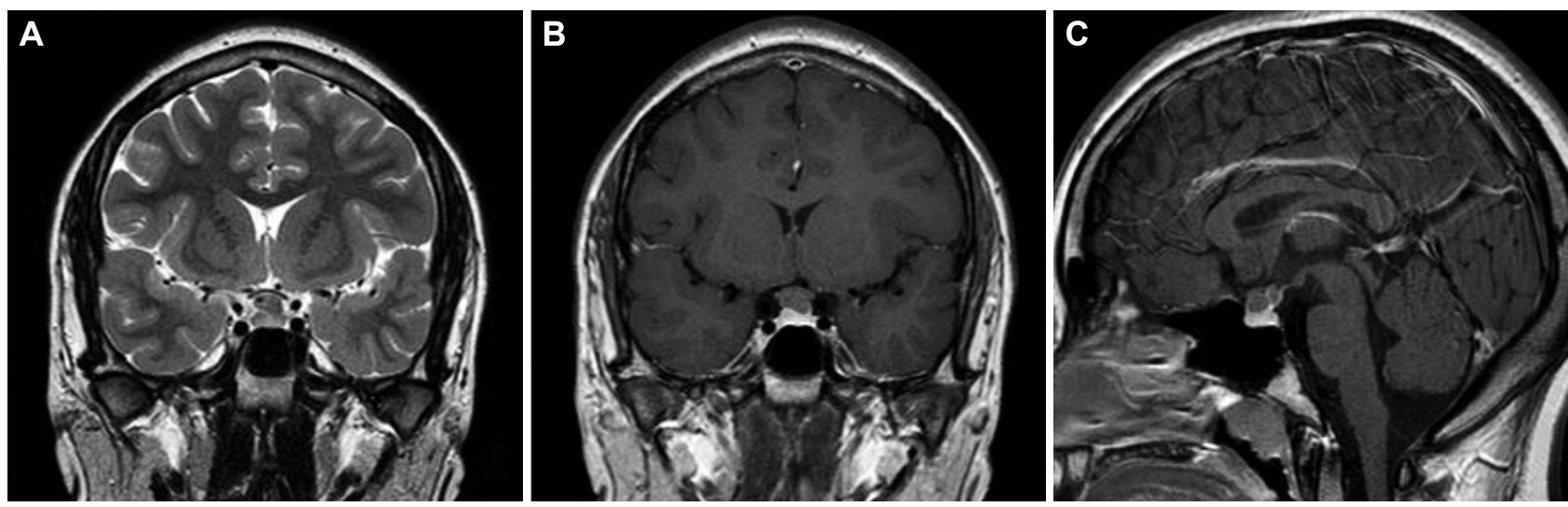

Fig. 1. A $12 \mathrm{~mm}$-sized tumor compressing the pituitary gland with suprasellar extension was found on T2-weighted image of brain MRI (A). Rim enhancement was observed on coronal view (B) as well as sagittal view of T1-enhanced image (C).
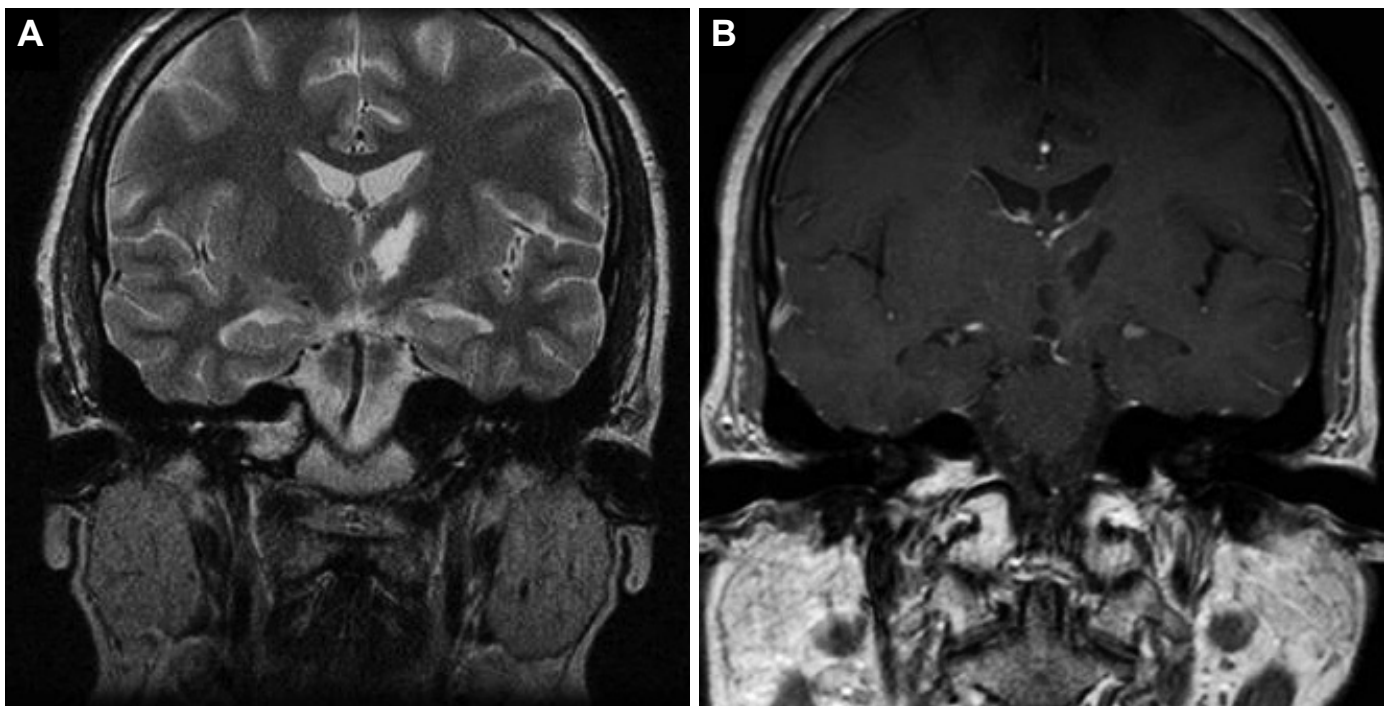

Fig. 2. T2-weighted image of brain MRI showed focal hyper signal intensity lesion in the anterior-superior-medial part of the left thalamus (A) without enhancement on T1-enhancement image (B), suggesting cerebral infarction as a sequelae of surgery after two months. 
tam $1000 \mathrm{mg} /$ day and lamotrigine $300 \mathrm{mg} /$ day were administered and there was no recurrence of generalized tonic-clonic seizures from August 2010 until August 2013. Myoclonic jerks including forced eye blinking recurred several times a year, albeit rarely.

In November 2013, when she was 20 years old, she admitted having excessive daytime sleepiness for the past 3 years. She denied having cataplexy, sleep paralysis or hypnagogic hallucinations. At the time, the antiepileptic drug regimen was not changed because the seizures were relatively well controlled. There were no focal signs on neurologic examination and laboratory findings were in the normal range. Follow up brain MRI showed the focal lesion in the anterior-superior-medial part of the left thalamus had decreased in the horizontal field but was slightly extended longitudinally. To eliminate the possibility of nonconvulsive seizures, video-EEG monitoring was performed, and did not show interictal or ictalepileptiform discharges. On the night of polysomnography (PSG) (Fig. 4), sleep latency was 3.5 minutes and total sleep time was 521 minutes with a nor- mal percentage (18.4\%) of REM stage sleep. Sleep architecture was normal and was composed of $6.1 \%$ of non-rapid eye movement (NREM) stage 1, $48.4 \%$ of NREM stage 2, 26.5\% of NREM stage 3 and $18.4 \%$ of REM stage sleep. REM sleep episodes occurred 5 times. The sleep onset REM period (SOREMP) was not observed. Sleep efficiency was about $98.7 \%$. A total of 10 episodes of hypopnea were recorded (apnea hypopnea index $=$ $1.2 / \mathrm{hr}$ ) and the respiratory disturbance index was $3.3 / \mathrm{h}$. On MSLT, mean sleep latency was 2.6 minutes and sleep-onset REM periods less than 15 minutes after sleep onset were detected in 3 of 5 nap trials. Based on the findings of both overnight PSG and MSLT, she was diagnosed with narcolepsy. Modafinil was prescribed with a dose up to $400 \mathrm{mg}$ /day, but was not effective in reducing excessive daytime sleepiness.

\section{DISCUSSION}

In our patient, excessive daytime sleepiness and/or daytime

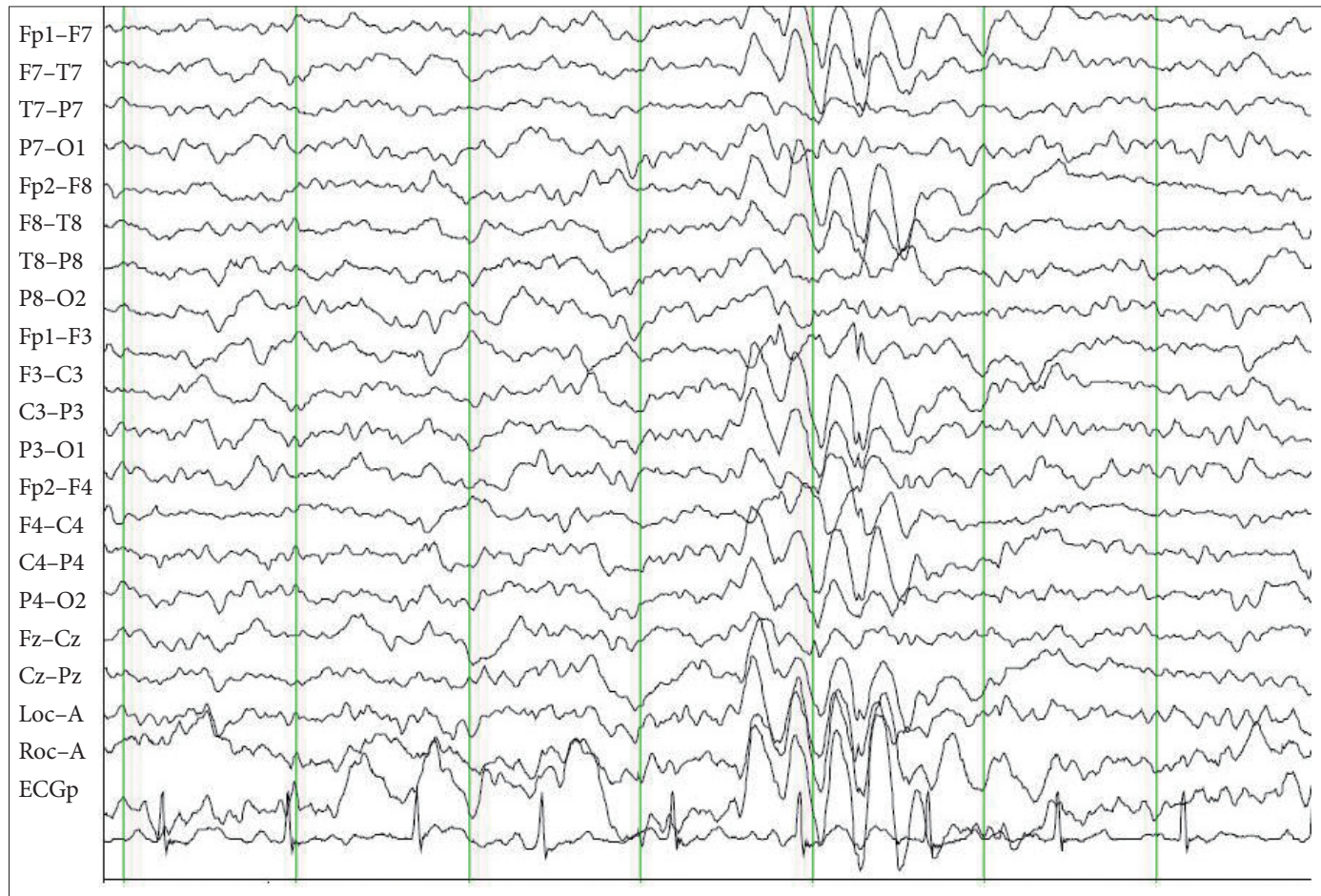

Fig. 3. Electroencephalography showed frequent frontal dominant 3-4 Hz generalized spike and wave complexes.

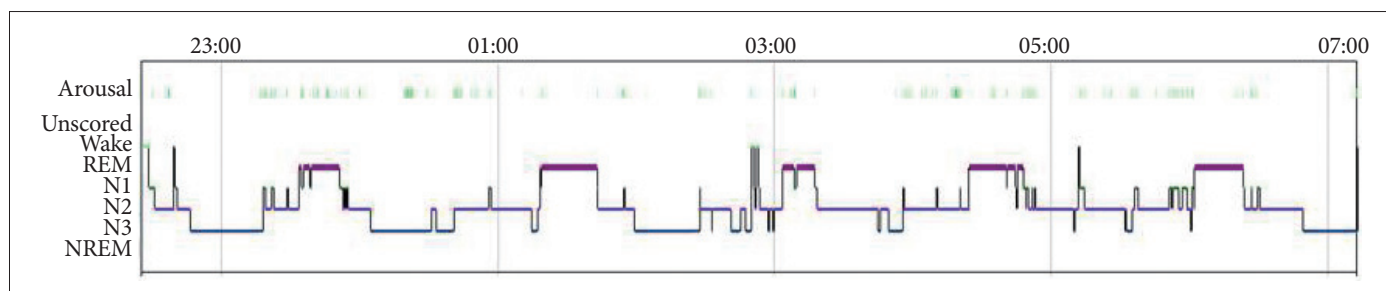

Fig. 4. Total sleep time was 521 minutes on polysomnography. Sleep architecture was normal and was composed of $6.1 \%$ of non-rapid eye movement (NREM) stage $1,48.4 \%$ of NREM stage 2, $26.5 \%$ of NREM stage 3, and $18.4 \%$ of rapid eye movement (REM) stage sleep. 
lapses into sleep appeared within two years after surgical removal of Rathke's cleft cyst. On MSLT, mean sleep latency was 2.6 minutes and SOREMPs within 15 minutes of sleep onset were recorded in three out of five nap trials. The preceding nocturnal PSG showed no sleep problems associated with daytime sleepiness. These findings are compatible with the current diagnostic criteria for narcolepsy without cataplexy based on the third edition of the International Classification of Sleep Medicine. ${ }^{6}$ To be diagnosed as symptomatic narcolepsy, the signs and symptoms of narcolepsy should be temporally associated with the underlying neurological process. Nishino and Kanbayashi ${ }^{3}$ suggested that narcolepsy onset should be within three years if the causative diseases are acute neurological conditions. Narcoleptic symptoms in our patient appeared two years after surgical removal of Rathke's cleft cyst. Therefore, the patient was diagnosed with symptomatic narcolepsy.

Considering that the Rathke's cleft cyst in this patient was relatively small (12 $\mathrm{mm}$ ) and excessive daytime sleepiness was absent before surgery but developed two years after surgery, her narcoleptic symptoms were attributed to a surgical complication from the Rathke's cleft cyst. Two months after surgery, brain MRI showed a focal hyper signal intensity lesion in the anterior-superior-medial part of the left thalamus, suggesting cerebral infarction as a sequelae of surgery. The present case may support the cases reported by Marcus et al. ${ }^{4}$ and Tachibana et al. ${ }^{5}$ regarding narcoleptic symptoms that became apparent after surgery on suprasellar tumors. In a similar report by Marcus et al. ${ }^{4}$ excessive daytime sleepiness appeared after two years postoperatively in a patient with suprasellar germinoma.

Similar to idiopathic narcolepsy with cataplexy, reduced CSF hypocretin levels are often seen in symptomatic narcolepsy with various etiologies. ${ }^{7}$ In contrast, a few symptomatic narcoleptic cases of paramedian thalamic infarctions have been reported with hypocretin levels within a normal range and impairment of hypocretin projection has been suggested to cause narcolepsy. ${ }^{8}$ The present case showed a vascular lesion in the left thalamus, but not in the hypothalamus, as a sequelae of surgery. Although CSF hypocretin level was not measured in the present case, our patient was thought to have functional impairment of hypocretin projection rather than damaged hypocretin-containing neurons as a result of surgery, resulting in symptomatic narcolepsy. Recently, Sakuta et al. ${ }^{9}$ performed MRI tractography on a patient with symptomatic narcolepsy following surgery for craniopharyngioma. They suggested that a lack of neuronal connections had contributed to the occurrence of symptomatic narcolepsy. In a literature review, Nishino and Kanbayashi ${ }^{3}$ reported that $55 \%$ of 33 symptomatic narcoleptic cases caused by brain tumors exhibited cataplexy, whereas the remaining cases had no evidence of cataplexy as in the present case.

Symptomatic narcolepsy associated with Rathke's cleft cyst was not found in the literature. Only one adult case with progressive hypersomnia was reported to be associated with Ra- thke's cleft cyst. ${ }^{10}$ PSG showed a prolonged total sleep period for 2 nights (523 and 590 minutes), with normal sleep architecture and the percentage of REM sleep at $35 \%$ and $24 \%$, respectively. The MSLT showed a mean latency of 5 minutes with dozing off during each session without REM sleep. He underwent surgery and the operative specimen revealed a Rathke's cleft cyst.

Our patient with Rathke's cleft cyst presented with generalized convulsive seizures prior to initial admission. However, seizures rarely occur in those with Rathke's cleft cyst or craniopharyngioma located in the suprasellar area. In a series of 66 pediatric patients with craniopharyngioma reported by Puget et al., ${ }^{11}$ seizures were the clinical features upon admission in $4.5 \%$. In contrast, seizures as a complication after the removal of craniopharyngioma are not rare. Zhang et al. ${ }^{12}$ found that seizures developed after surgery in $9.4 \%$ of 202 patients with craniopharyngioma.

In the present case, myoclonic jerks in the proximal extremities were absent before surgery, but developed within four months after surgery. Loss of consciousness sometimes occurred with generalized tonic-clonic seizures. EEG showed frequent frontal dominant 3-4 Hz spike and wave complexes (Fig. 3). These recurrent seizures were thought to be caused by a left thalamic lesion that was a complication of the surgery. There are several reports on unilateral thalamic lesions associated with epileptic seizures which showed generalized spike-waves discharges or continuous spike-waves during slow wave sleep. ${ }^{13}$ Kelemen et al. ${ }^{14}$ reported a patient who was initially diagnosed with idiopathic generalized epilepsy but was eventually diagnosed three years later as secondary generalized epilepsy with generalized spike-wave patterns associated with a right thalamic tumor.

In summary, we report a woman with symptomatic narcolepsy that developed after surgical removal of Rathke's cleft cyst, which has never before been reported as a causative factor for symptomatic narcolepsy. In addition, myoclonic and/or generalized tonic-clonic seizures were associated with a left thalamic lesion generated by a complication of surgery.

\section{Conflicts of Interest}

The authors have no financial conflicts of interest.

\section{REFERENCES}

1. Kubota H, Kanbayashi T, Tanabe Y, Ito M, Takanashi J, Kohno Y, et al. Decreased cerebrospinal fluid hypocretin-1 levels near the onset of narcolepsy in 2 prepubertal children. Sleep 2003;26:555-7.

2. Mignot E, Hayduk R, Black J, Grumet FC, Guilleminault C. HLA DQB1*0602 is associated with cataplexy in 509 narcoleptic patients. Sleep 1997;20:1012-20.

3. Nishino S, Kanbayashi T. Symptomatic narcolepsy, cataplexy and hypersomnia, and their implications in the hypothalamic hypocretin/ orexin system. Sleep Med Rev 2005;9:269-310.

4. Marcus CL, Trescher WH, Halbower AC, Lutz J. Secondary narcolepsy in children with brain tumors. Sleep 2002;25:435-9.

5. Tachibana N, Taniike M, Okinaga T, Ripley B, Mignot E, Nishino S. Hypersomnolence and increased REM sleep with low cerebrospinal fluid hypocretin level in a patient after removal of craniopharyngioma. 
Sleep Med 2005;6:567-9.

6. American Academy of Sleep Medicine International Classification of Sleep Disorders. 3rd ed. Westchester, IL: American Academy of Sleep Medicine 2014

7. Nokura K, Kanbayashi T, Ozeki T, Koga H, Zettsu T, Yamamoto H, et al. Hypersomnia, asterixis and cataplexy in association with orexin Areduced hypothalamic tumor. J Neurol 2004;251:1534-5.

8. Tohyama J, Kanazawa O, Akasaka N, Kamimura T. [A case of bilateral paramedian thalamic infarction in childhood with the sensory disturbance and the sensory loss of taste]. No To Hattatsu 2004;36:65-9.

9. Sakuta K, Nakamura M, Komada Y, Yamada S, Kawana F, Kanbayashi $\mathrm{T}$, et al. Possible mechanism of secondary narcolepsy with a long sleep time following surgery for craniopharyngioma. Intern Med 2012;51: 413-7.

10. Autret A, Lucas B, Mondon K, Hommet C, Corcia P, Saudeau D, et al. Sleep and brain lesions: a critical review of the literature and additional new cases. Neurophysiol Clin 2001;31:356-75.

11. Puget S, Garnett M, Wray A, Grill J, Habrand JL, Bodaert N, et al. Pediatric craniopharyngiomas: classification and treatment according to the degree of hypothalamic involvement. J Neurosurg 2007;106(1 Suppl): 3-12.

12. Zhang YQ, Ma ZY, Wu ZB, Luo SQ, Wang ZC. Radical resection of 202 pediatric craniopharyngiomas with special reference to the surgical approaches and hypothalamic protection. Pediatr Neurosurg 2008;44: 435-43.

13. Inghilleri M, Clemenzi A, Conte A, Frasca V, Manfredi M. Bilateral spike-and-wave discharges in a hemi-deafferented cortex. Clin Neurophysiol 2002;113:1970-2.

14. Kelemen A, Barsi P, Gyorsok Z, Sarac J, Szucs A, Halász P. Thalamic lesion and epilepsy with generalized seizures, ESES and spike-wave paroxysms--report of three cases. Seizure 2006;15:454-8. 